\title{
Increased risk of tinnitus in patients with early-onset cataracts: a nationwide population-based case-control study
}

\author{
Yi-Ching Hsieh ${ }^{1}$, Cheng-Hsien Chang ${ }^{1}$, Yi-Yu Tsai ${ }^{1,2}$, Cheng-Li Lin ${ }^{3,4}$, Telk-Ying Ng ${ }^{5}$ and Chun-Chi Chiang ${ }^{1,2^{*}}$
}

\begin{abstract}
Background: This study aimed to investigate the association between early-onset cataract and tinnitus using a population-based database.

Methods: Retrospective claims data from the Taiwan National Health Insurance Research Database were analysed. Study subjects comprised patients with early-onset cataract, aged 20-55 years and diagnosed between 2000 and $2010(n=2084)$ and a comparison cohort without the disease $(n=8336)$. Both cohorts were followed until 2010 to estimate the incidence of tinnitus. To calculate the risk of tinnitus in the case and control groups, Cox proportional hazards models were used and presented as hazard ratios (HRs), adjusted HRs (aHRs) and 95\% confidence intervals (Cls).

Results: Patients with early-onset cataract had 1.53-fold increased risk ( $\mathrm{HR}=1.53,95 \% \mathrm{Cl}=1.17-2.01, p<0.01)$ of developing tinnitus than controls. The number of patients with vertigo $(p<0.0001)$, insomnia $(p<0.0001)$, anxiety $(p<0.0001)$ and hearing loss $(p<0.0001)$ as comorbidities was also significantly higher in the case group. After adjusting for age, sex and all listed comorbidities, patients with increasing age (aHR $=1.04,95 \% \mathrm{Cl}=1.02-1.07)$, early-onset cataract $(\mathrm{aHR}=1.32,95 \% \mathrm{Cl}=1.01-1.74)$, vertigo $(\mathrm{aHR}=1.75,95 \% \mathrm{Cl}=1.15-2.67)$, insomnia $(\mathrm{aHR}=1.48$, $95 \% \mathrm{Cl}=1.14-1.93)$ and hearing loss $(a \mathrm{HR}=6.20,95 \% \mathrm{Cl}=3.58-10.70)$ had significantly higher risk of tinnitus.

Conclusions: Patients with early-onset cataract are at an increased risk of developing tinnitus in subsequent years and should receive further evaluation for early diagnosis and management if any signs of tinnitus occur.
\end{abstract}

Keywords: Early-onset cataract, Tinnitus, Cohort study, Case-control, Risk

\section{Background}

Cataract is defined as the opacity of the eye lens. It is also the leading cause of blindness, accounting for $51 \%$ of cases of blindness globally according to the latest assessment (2010) [1]. The opacity of the lens directly results from oxidative stress [2]. Oxidative stress is an imbalance between the rate of oxidant production and the rate of its degradation. The loss of glutathione

\footnotetext{
* Correspondence: elsa10019@yahoo.com.tw

'Department of Ophthalmology, China Medical University Hospital, China

Medical University, No. 2, Yude Road, Taichung, Taiwan

${ }^{2}$ School of Medicine, China Medial University, Taichung, Taiwan

Full list of author information is available at the end of the article
}

(GSH), the primary and essential lenticular antioxidant, is a crucial feature in age-related nuclear cataract [3]. Age-related cataracts usually develop in patients aged > 60 years; however, cataracts may also develop in some patients aged $<55$ years [4]. Multiple risk factors are responsible for the development of early-onset cataract, such as diabetes, high myopia, steroid use, smoking and ultraviolet (UV) light exposure [5-9]. Oxidative stress may be one of the major contributing factors in earlyonset cataract. Other pathogeneses of early-onset cataract include congenital, idiopathic, chronic inflammation, traumatic and cataract related to systemic diseases.

(c) The Author(s). 2020 Open Access This article is licensed under a Creative Commons Attribution 4.0 International License, which permits use, sharing, adaptation, distribution and reproduction in any medium or format, as long as you give appropriate credit to the original author(s) and the source, provide a link to the Creative Commons licence, and indicate if changes were made. The images or other third party material in this article are included in the article's Creative Commons licence, unless indicated otherwise in a credit line to the material. If material is not included in the article's Creative Commons licence and your intended use is not permitted by statutory regulation or exceeds the permitted use, you will need to obtain permission directly from the copyright holder. To view a copy of this licence, visit http://creativecommons.org/licenses/by/4.0/ The Creative Commons Public Domain Dedication waiver (http://creativecommons.org/publicdomain/zero/1.0/) applies to the data made available in this article, unless otherwise stated in a credit line to the data. 
Tinnitus, defined as the subjective perception of noises in the absence of an external auditory stimulus, is a multifactorial disorder. The reported prevalence of tinnitus in the general population is approximately 10 to $15 \%$ [10]. There are several causes of tinnitus, including otologic, neurologic and infectious causes $[11,12]$. Hearing loss has been considered the most common cause [13]. Moreover, several authors have observed that reactive oxygen species (ROS) plays a role in the pathogenesis of tinnitus [14-16].

Because oxidative stress is related to both early-onset cataract and tinnitus, the present study investigates whether patients with early-onset cataracts are at risk of tinnitus using the nationwide population-based dataset from Taiwan.

\section{Methods}

\section{Data source}

Taiwan established a nationwide database, the National Health Insurance Research Database (NHIRD), in 1995. Presently, this database contained comprehensive medical information of $>99 \%$ of single-payer insurance beneficiaries in Taiwan. The medical information included the historical record of out- and inpatients and medications but without identification to protect the privacy of each subject.

In this study, we used the Longitudinal Health Insurance Database, which selected one million subjects from the NHIRD by randomization.

All previous diagnoses in the database were coded according to the International Classification of Disease, Ninth Revision, Clinical Modification (ICD-9-CM). The Research Ethics Committee of China Medical University and Hospital in Taiwan approved the study protocol (CMUH-104-REC2-115-R3).

\section{Study population}

Based on the study objective, we aimed to confirm the association between early-onset cataract and tinnitus. We selected patients with early-onset cataract (ICD-9CM: 366.00, 366.01, 366.02, 366.03, 366.04, 366.09, 366.17 and 366.18 ) as the case group and defined the diagnosis date as the index year; the control group was selected by 4:1 matching with the case group, and the matching variables included age, sex and index year. The enrollment date for the subject in the control group was matched with the same year of the subject in the earlyonset cataract cohort, while the month and the day was randomly assigned. The study population was followed until the development of tinnitus, lost to follow-up or 31 December 2011 and the study period was from 2000 to 2011. A total of 2193 patients aged 20-55 years with newly-diagnosed early-onset cataract were identified in our study. Patients those with a history of tinnitus (ICD-
9-CM: 388.3) $(n=109)$ before the index date were excluded from our study.

The comorbidities evaluated in this study were vertigo (ICD-9-CM: 386), insomnia (ICD-9-CM: 780), anxiety (ICD-9-CM: 300.00) and hearing loss (ICD-9-CM: 388389).

\section{Statistical analysis}

This study included demographic, residential and comorbidities variables. The continuous and categorical variables were presented as mean \pm standard deviation and number (\%), respectively. To compare the difference of each variable in the two groups, $t$-test and chi-square test were used, respectively. To calculate the risk of tinnitus in the case and control groups, Cox proportional hazards models were used and presented as hazard ratios (HRs), adjusted HRs (aHRs) and 95\% confidence intervals (CIs). The cumulative incidence of tinnitus in the two groups was obtained using the Kaplan-Meier method, and the log-rank test was used to compare the cumulative incidences in the two groups. All statistical analyses were performed using SAS version 9.4 (SAS Institute Inc., Cary, NC). The cumulative incidence curve was plotted using $\mathrm{R}$ software. The significance level was set at a two-sided $P$-value $<0.05$.

\section{Results}

There were a total of 10,420 eligible participants in this study: 2084 patients with early-onset cataract (case) and 8336 individuals without early-onset cataract (control) (Table 1). In this study, $50.1 \%$ of the study subjects were men, and the mean age in the case and control groups was 46.3 and 45.4 years, respectively; most of them were aged between 46 and 55 years $(67.0 \%)$ and living in the highest urbanization level region. There were no significant differences in sex, age and index year between the two groups. The number of patients with vertigo $(p<$ $0.0001)$, insomnia $(\mathrm{p}<0.0001)$, anxiety $(\mathrm{p}<0.0001)$ and hearing loss $(\mathrm{p}<0.0001)$ as comorbidities was significantly higher in the case group. The mean follow up years of the early-onset cataract cohort is $4.76( \pm \mathrm{SD}$ 3.12 ) years and of the non-early-onset cataract cohort is 4.82 ( \pm SD 3.17 ) years.

Table 2 presents the incidence of tinnitus and HR between the case and control groups after stratification by demographic characteristics and comorbidities. The incidence rate of tinnitus in population without early-onset cataract was 4.83 (per 1000 person-years), and 7.36 in population with early-onset cataract. Patients with earlyonset cataract had 1.53-fold increased risk $(\mathrm{HR}=1.53$, $95 \% \mathrm{CI}=1.17-2.01$ ) of developing tinnitus than controls. Considering sex, stratified age, comorbidities and followup duration, patients with early-onset cataract who were women $(\mathrm{HR}=1.43,95 \% \mathrm{CI}=1.00-2.05)$, men $(\mathrm{HR}=$ 
Table 1 Comparisons in demographic characteristics and comorbidities in patient with and without Early-onset cataract

\begin{tabular}{|c|c|c|c|}
\hline & \multicolumn{2}{|c|}{ Early-onset cataract } & \multirow[t]{3}{*}{$p$-value } \\
\hline & No & Yes & \\
\hline & $(N=8336)$ & $(N=2084)$ & \\
\hline \multicolumn{3}{|l|}{ Gender } & \multirow[t]{3}{*}{0.99} \\
\hline Women & $4160(49.9)$ & $1040(49.9)$ & \\
\hline Men & $4176(50.1)$ & $1044(50.1)$ & \\
\hline \multicolumn{3}{|l|}{ Age stratified } & \multirow[t]{4}{*}{0.99} \\
\hline$\leq 35$ & $772(9.26)$ & $193(9.26)$ & \\
\hline $36-45$ & $1980(23.8)$ & $495(23.8)$ & \\
\hline $46-55$ & $5584(67.0)$ & $1396(67.0)$ & \\
\hline Age, mean $\pm \mathrm{SD}^{\mathrm{a}}$ & $45.4(7.43)$ & $46.3(7.41)$ & $<0.0001$ \\
\hline \multicolumn{3}{|l|}{ Urbanization level $^{\dagger}$} & \multirow[t]{5}{*}{0.02} \\
\hline 1 (highest) & $2601(31.2)$ & $670(32.2)$ & \\
\hline 2 & 2558 (30.7) & $682(32.7)$ & \\
\hline 3 & 1558 (18.7) & $329(15.8)$ & \\
\hline 4 (lowest) & 1619 (19.4) & $403(19.3)$ & \\
\hline \multicolumn{4}{|l|}{ Comorbidity } \\
\hline Vertigo & $358(4.29)$ & $132(6.33)$ & $<0.0001$ \\
\hline Insomnia & 2689 (32.3) & $961(46.1)$ & $<0.0001$ \\
\hline Anxiety & $589(7.07)$ & $222(10.7)$ & $<0.0001$ \\
\hline Hearing loss & $45(0.54)$ & $28(1.34)$ & $<0.0001$ \\
\hline
\end{tabular}

Chi-Square Test, ${ }^{a}$ t-test

${ }^{\dagger}$ The urbanization level was categorized by the population density of the residential area into 4 levels, with level 1 as the most urbanized and level 4 as the least urbanized
$1.68,95 \% \mathrm{CI}=1.11-2.53)$, aged $<35$ years $(\mathrm{HR}=7.97$, $95 \% \mathrm{CI}=2.40-26.5)$ and aged between 36 and 45 years $(\mathrm{HR}=2.50,95 \% \mathrm{CI}=1.34-4.66)$, had no comorbidities $(\mathrm{HR}=1.63,95 \% \mathrm{CI}=1.09-2.44)$ and had a follow-up duration of $>4$ years $(\mathrm{HR}=1.70,95 \% \mathrm{CI}=1.13-2.54)$ had significantly higher risk of developing tinnitus than controls. After adjusting for age, sex and comorbidities of vertigo, insomnia, anxiety and hearing loss, patients with early-onset cataract $(\mathrm{aHR}=1.32,95 \% \mathrm{CI}=1.00$ $1.73)$, aged $<35$ years $(\mathrm{aHR}=6.04,95 \% \mathrm{CI}=1.74-21.0)$, aged between 36 and 45 years $(\mathrm{aHR}=2.46,95 \% \mathrm{CI}=$ 1.31-4.61), without comorbidities $(\mathrm{aHR}=1.58,95 \% \mathrm{CI}=$ 1.05-2.37) and followed for > 4 years $(\mathrm{aHR}=1.62$, 95\% $\mathrm{CI}=1.08-2.44)$ had higher risk of developing tinnitus than controls. Figure 1 shows that the cumulative incidence of tinnitus was significantly higher in patients with early-onset cataract than in controls $(p=0.002)$.

Table 3 presents the risk factors of tinnitus. Study subjects who were women $(\mathrm{HR}=1.39,95 \% \mathrm{CI}=1.09-1.77)$ and had increasing age $(\mathrm{HR}=1.05,95 \% \mathrm{CI}=1.03-1.08)$, early-onset cataract $(\mathrm{HR}=1.53,95 \% \mathrm{CI}=1.17-2.01)$, vertigo $(\mathrm{HR}=2.62,95 \% \mathrm{CI}=1.75-3.93)$, insomnia $(\mathrm{HR}=$ $1.94,95 \% \mathrm{CI}=1.52-2.47)$, anxiety $(\mathrm{HR}=1.77,95 \% \mathrm{CI}=$ 1.19-2.63) and hearing loss $(\mathrm{HR}=8.75,95 \% \mathrm{CI}=5.11-$ 15.0) had significantly higher risk of developing tinnitus. After adjusting for age, sex and all listed comorbidities, patients with increasing age $(\mathrm{aHR}=1.04,95 \% \mathrm{CI}=1.02-$ 1.07), early-onset cataract $(\mathrm{aHR}=1.32,95 \% \mathrm{CI}=1.01-$ 1.74 ), vertigo $(\mathrm{aHR}=1.75,95 \% \mathrm{CI}=1.15-2.67)$, insomnia $(\mathrm{aHR}=1.48, \quad 95 \% \quad \mathrm{CI}=1.14-1.93)$ and hearing loss

Table 2 Comparison of incidence densities of Tinnitus and hazard ratio between with and without Early-onset cataract by demographic characteristics and comorbidity

\begin{tabular}{|c|c|c|c|c|c|c|c|c|}
\hline & \multicolumn{6}{|c|}{ Early-onset cataract } & \multirow[b]{3}{*}{ Crude HR* $(95 \% \mathrm{Cl})$} & \multirow[b]{3}{*}{ Adjusted $\mathrm{HR}^{\dagger}(95 \% \mathrm{Cl})$} \\
\hline & \multicolumn{3}{|l|}{ No } & \multicolumn{3}{|l|}{ Yes } & & \\
\hline & Event & PY & Rate $^{\#}$ & Event & PY & Rate $^{\#}$ & & \\
\hline All & 194 & 40,186 & 4.83 & 73 & 9913 & 7.36 & $1.53(1.17,2.01)^{* *}$ & $1.32(1.00,1.73)^{*}$ \\
\hline \multicolumn{9}{|l|}{ Gender } \\
\hline Women & 116 & 20,373 & 5.69 & 41 & 5043 & 8.13 & $1.43(1.00,2.05)^{*}$ & $1.25(0.87,1.79)$ \\
\hline Men & 78 & 19,813 & 3.94 & 32 & 4869 & 6.57 & $1.68(1.11,2.53)^{*}$ & $1.41(0.93,2.15)$ \\
\hline \multicolumn{9}{|l|}{ Stratify age } \\
\hline$\leq 35$ & 4 & 3872 & 1.03 & 8 & 966 & 8.28 & $7.97(2.40,26.5)^{* * *}$ & $6.04(1.74,21.0)^{* *}$ \\
\hline $36-45$ & 26 & 9493 & 2.74 & 16 & 2341 & 6.84 & $2.50(1.34,4.66)^{* *}$ & $2.46(1.31,4.61)^{* *}$ \\
\hline $46-55$ & 164 & 26,821 & 6.11 & 49 & 6606 & 7.42 & $1.22(0.89,1.68)$ & $1.09(0.79,1.50)$ \\
\hline \multicolumn{9}{|l|}{ Comorbidity $^{\ddagger}$} \\
\hline No & 96 & 27,707 & 3.46 & 31 & 5476 & 5.66 & $1.63(1.09,2.44)^{*}$ & $1.58(1.05,2.37)^{*}$ \\
\hline Yes & 98 & 12,479 & 7.85 & 42 & 4436 & 9.47 & $1.21(0.84,1.73)$ & $1.20(0.84,1.73)$ \\
\hline \multicolumn{9}{|c|}{ Follow-up time $e^{\ddagger}$} \\
\hline$\leq 4$ years & 113 & 25,582 & 4.42 & 40 & 6404 & 6.25 & $1.42(0.99,2.03)$ & $1.13(0.78,1.63)$ \\
\hline$>4$ years & 81 & 14,604 & 5.55 & 33 & 3509 & 9.40 & $1.70(1.13,2.54)^{*}$ & $1.62(1.08,2.44)^{*}$ \\
\hline
\end{tabular}

Rate ${ }^{\#}$, incidence rate, per 1000 person-years; Crude $\mathrm{HR}^{*}$, relative hazard ratio; Adjusted $\mathrm{HR}^{\dagger}$ : multivariable analysis including age, gender, and comorbidities of vertigo, insomnia, anxiety, and hearing loss; ${ }^{*} p<0.05,{ }^{* *} p<0.01$, ${ }^{* * *} p<0.001$

Comorbidity : Patients with any one of the comorbidities vertigo, insomnia, anxiety, and hearing loss were classified as the comorbidity group

${ }^{\ddagger}$ The follow-up time is partitioned into 2 segments (years $\leq 4$, and $>4$ years) by median 


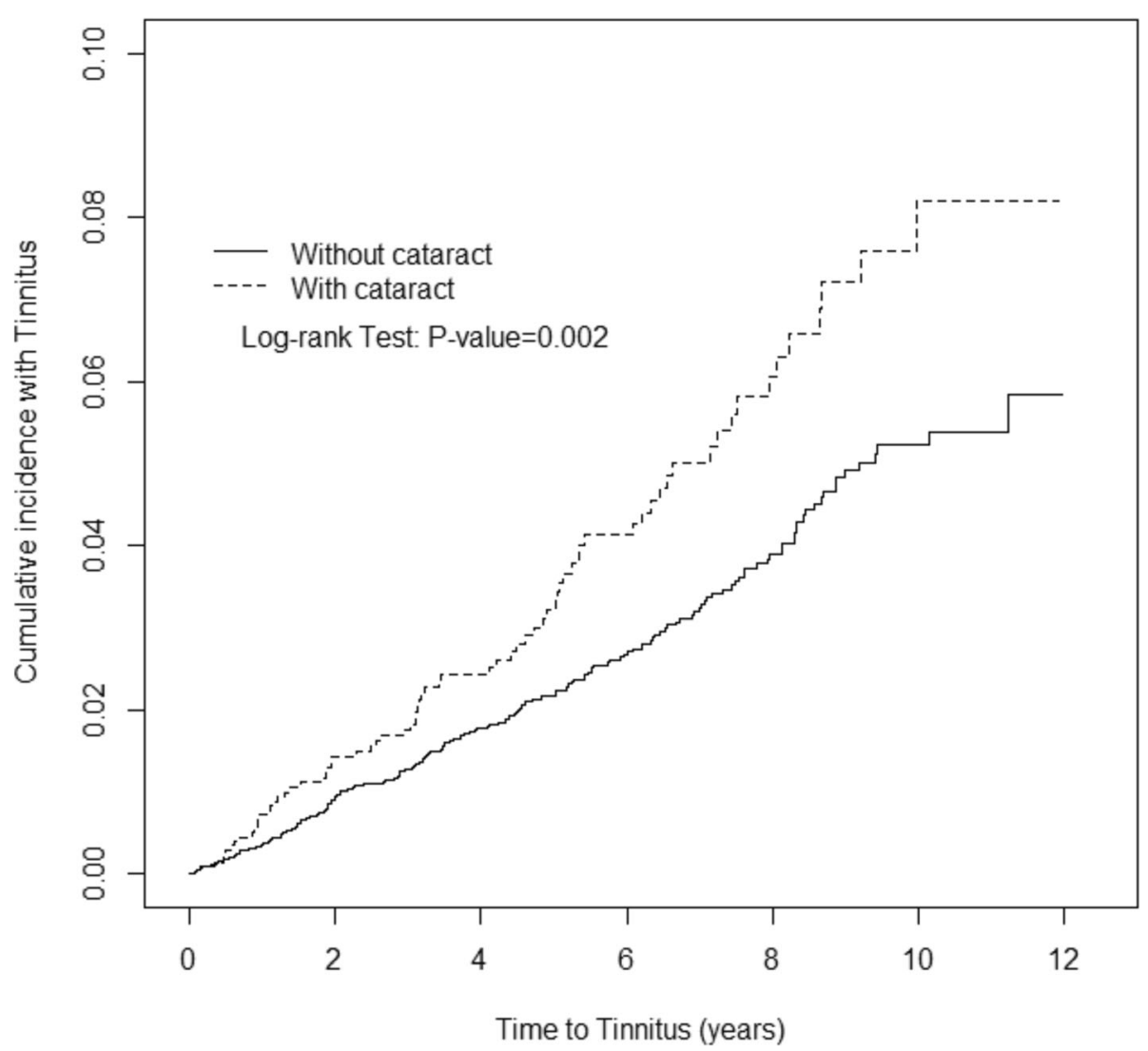

Fig. 1 Cumulative incidence of Tinnitus for patients with (dashed line) or without (solid line) Early-onset cataract

Table 3 Cox model with hazard ratios and 95\% confidence intervals of Tinnitus associated with Early-onset cataract and covariates

\begin{tabular}{|c|c|c|c|c|}
\hline \multirow[b]{2}{*}{ Variable } & \multicolumn{2}{|c|}{ Crude } & \multicolumn{2}{|c|}{ Adjusted $^{\dagger}$} \\
\hline & $\mathrm{HR}$ & $(95 \% \mathrm{Cl})$ & $\mathrm{HR}$ & $(95 \% \mathrm{Cl})$ \\
\hline \multicolumn{5}{|l|}{ Gender } \\
\hline Women & 1.39 & $(1.09,1.77)^{* *}$ & 1.22 & $(0.95,1.57)$ \\
\hline Men & 1 & (reference) & 1 & (reference) \\
\hline Age, years & 1.05 & $(1.03,1.08)^{* * *}$ & 1.04 & $(1.02,1.07)^{* * *}$ \\
\hline \multicolumn{5}{|l|}{ Urbanization level } \\
\hline 1 (highest) & 1.02 & $(0.71,1.47)$ & - & - \\
\hline 2 & 1.27 & $(0.90,1.81)$ & - & - \\
\hline 3 & 1.09 & $(0.73,1.64)$ & - & - \\
\hline 4 (lowest) & 1 & (reference) & - & - \\
\hline \multicolumn{5}{|c|}{ Baseline comorbidities (yes vs no) } \\
\hline Early-onset cataract & 1.53 & $(1.17,2.01)^{* *}$ & 1.32 & $(1.01,1.74)^{*}$ \\
\hline Vertigo & 2.62 & $(1.75,3.93)^{* * * *}$ & 1.75 & $(1.15,2.67)^{* *}$ \\
\hline Insomnia & 1.94 & $(1.52,2.47)^{* * *}$ & 1.48 & $(1.14,1.93)^{* *}$ \\
\hline Anxiety & 1.77 & $(1.19,2.63)^{* *}$ & 1.22 & $(0.80,1.84)$ \\
\hline Hearing loss & 8.75 & $(5.11,15.0)^{* * *}$ & 6.20 & $(3.58,10.7)^{* * * *}$ \\
\hline
\end{tabular}

Crude $\mathrm{HR}^{*}$, relative hazard ratio; Adjusted $\mathrm{HR}^{+}$: multivariable analysis including age, gender, and comorbidities of vertigo, insomnia, anxiety, and hearing loss ${ }^{*} p<0.05,{ }^{* *} p<0.01,{ }^{* * *} p<0.001$
$(\mathrm{aHR}=6.20,95 \% \mathrm{CI}=3.58-10.70)$ still had significantly higher risk of developing tinnitus.

\section{Discussion}

The population aged $>55$ years is more susceptible to lens opacity [17], and the prevalence of tinnitus is also increasing with age [18]. However, no previous study has demonstrated the relationship between early-onset cataract and the risk of tinnitus. The present retrospective cohort study found that the incidence rate of tinnitus in population without early-onset cataract was 4.83 (per 1000 person-years), and 7.36 in population with earlyonset cataract. Moreover, the risk of tinnitus was 1.53fold higher in patients with early-onset cataract than controls. In the category less than 4 years of follow-up, the incidence of tinnitus and hazard ratio between population with and without early-onset cataract demonstrated no statistical difference. The follow-up duration may not be long enough to detect whether early-onset cataract is a risk factor for tinnitus. In the other category with no statistical difference, age $46-55$, the reason may be related to the age distribution of cataract and tinnitus. The prevalence of cataract increased with age and most occurred after age of 50 years, as well as tinnitus. As such, early-onset cataract may not be shown as a 
significant risk factor for tinnitus in the age group older than 50 years.

The number of patients with vertigo $(p<0.0001)$, insomnia $(p<0.0001)$, anxiety $(p<0.0001)$ and hearing loss $(p<0.0001)$ as comorbidities was significantly higher in patients with early-onset cataract than in controls. Insomnia, hearing loss and anxiety are common symptoms that are associated with tinnitus, and these symptoms are highly relevant to tinnitus severity $[12,19,20]$. In contrast, cataracts is not well-known associated with insomnia, anxiety and hearing loss.. Therefore, we select these associating factors to evaluate further correlation between early-onset cataract and tinnitus. In addition to our findings, there were few reports detecting the correlation between insomnia, anxiety, hearing loss and cataract respectively. The rate of anxiety of cataract patients is $18.0 \%$ in a Chinese-based study, which is in keep with cataract patients in Russia (20\%), higher than the rate $(7.0 \%)$ in healthy people reportedly $[21,22]$. The BEAVER DAM study revealed that the combinations of cataract and hearing loss were common, occurring in $28 \%$ of the population $48-92$ years, increasing consistently with age. In younger population (48-59 years), the prevalence of simultaneous cataract and hearing loss was $3.1 \%$. Although risk is strongly related to age, some elective exposures appear to influence risk, such as heavy drinking and smoking [23]. The association between sleep and cataract is based on circadian rhythms. Cataract is opacity of the lens characterized by light scattering and absorption, especially the blue light. Thus, cataract may result in a decreased potential for circadian photoentrainment via retinohypothalamic tract, which also regulates the sleeping pattern. Decreased transmission of blue light to the retina in the opacified lens is significantly associated with an increased risk of sleep disturbances [24]. Alexander I et al. found that overall sleep quality and sleep latency of the patients improves after removal of cataract irrespective of the type of IOL implanted [25].

To further investigate the relationship between earlyonset cataract and comorbidities of tinnitus, we analysed the risk factors of tinnitus based on the data in this large population-based study. After adjusting for possible confounding factors, patients with early-onset cataract still had significant higher risk of tinnitus, similar to those with increasing age, vertigo, insomnia and hearing loss.

Several possible correlations exist between early-onset cataract and tinnitus. Oxidative stress associated with UV light exposure plays an important role in the pathogenesis of cataracts. The loss of GSH, the essential and primary lenticular antioxidant, from the nuclear region is probably the crucial feature that precedes cataract formation [26]. Depletion of GSH allows low levels of oxidant to damage epithelial $\mathrm{Na} / \mathrm{K}$-ATPase and proteins associated with cell membrane permeability. Additionally, Boscia et al. [27] found that the ROS might initiate a cascade of toxic biochemical reactions such as peroxidation of the membrane lipids, which causes intracellular protein aggregation and precipitation, resulting in lens opacification. In tinnitus, high concentrations of ROS can have cytotoxic and neurotoxic effects, causing damage to the auditory hair cells of the labyrinth and acoustic system [28]. Neri et al. [29] reported that the levels of oxidative damage markers (malondialdehyde, 4hydroxynonenal, myeloperoxidase) were higher in patients with tinnitus than in controls, whereas GSH peroxidase, an antioxidant enzyme, had lower activity in patients with tinnitus, sharing a similar pathogenesis with cataract.

Metabolic disorder, such as diabetes mellitus (DM), is another risk factor of early-onset cataract. In patients with DM, glucosylation of lens crystallins contributes to the accumulation of glycation end products, causing aggregation of high-molecular-weight material and resulting in lens opacification [30]. Studies have demonstrated that hyperglycaemia is associated with the impairment of cochlear microcirculation, especially in the stria vascularis. Ischaemia and hypoxia result in loss of outer hair cells and damage of neural units, leading to inner ear disorders, including tinnitus [31, 32]. In addition to DM, smoking is also a risk factor of both early-onset cataract and tinnitus $[18,33,34]$. Similar to the mechanism in $\mathrm{DM}$, smoking-mediated vascular dysfunction and arteriosclerosis contribute to ischaemia and hypoxia in cochlear microcirculation [35]. Moreover, hydrogen cyanide, lead, styrene and toluene in cigarette are ototoxic agents [36]. Cyanide is also responsible for the development of early-onset cataract [37]. The effect of cyanide toxicity on $\mathrm{Ca}^{2+}$-ATPase activities may result in the disruption of vitreous and lenticular calcium homeostasis, causing $\mathrm{Ca}^{2+}$ accumulation and lenticular opacification [38].

Occupational exposure, including ionizing radiation exposure (radiologic technologists) and solar radiation exposure (outdoor worker), is a risk factor for cataract $[39,40]$. In addition, excessive fuel exposure (solid cooking fuel combustion and poor kitchen ventilation) and metallic exposure (heavy metals work) are other possible risk factors associated with occupation [34, 41]. There are few epidemiological studies estimating occupational risk for tinnitus. Prevalence for working in noisy environment for $>10$ years was estimated higher in both men and women in comparison with those with no occupational exposure to noise [42]. Engdahl B et al. found that occupation had significant effects on the prevalence of tinnitus [43]. Crane and hoist operators and miners in men, and laboratory assistants in women had the highest tinnitus prevalence. In women, occupations with the 
highest risks were not typically noisy ones, and housekeeper and the group of occupationally inactive also had higher prevalence. There might be an association between fuel exposure and tinnitus, like in cataract. No definite common occupational exposure exists in earlyonset cataract and tinnitus in literature. Further research providing a better evaluation of occupation as risk factor is needed.

Steroids use is one of the cause of early-onset cataract. Nevertheless, no studies advocate long-term use of steroids in current management of tinnitus. There are no licensed drugs in Europe or North America for treatment of spontaneous idiopathic tinnitus [20]. Drugs from broad categories have been tested for effect on tinnitus; however, there is no standard treatments. For tinnitus with idiopathic sudden sensorineural hearing loss, intratympanic dexamethasone treatment has been showed effective in short term [44]. The other study demonstrated intratympanic drug therapy with steroid has resulted in both short- and long-term tinnitus relief in 7 of 10 patients $(70 \%)$ identified to have a predominantly cochlear-type tinnitus [45]. However, there was no reports about benefit of long-term use of steroids for tinnitus. As such, steroids use may not be responsible for the correlation between early-onset cataract and tinnitus.

There are few limitations in this study. First, the diagnoses of early-onset cataract, tinnitus and comorbidities are completely dependent on ICD codes. However, the reliability is strengthened by the NHI Bureau of Taiwan. Hospitals receive heavy penalties from the NHI Bureau when overcharging, discrepancies and malpractice are detected. Second, this study is an observational retrospective cohort study, so a small number of patients may have undetected early-onset cataract or tinnitus and might have had a possibility of being categorised in the wrong cohort consequently. Nevertheless, the sample sizes are large in both the case and control groups, reducing the selection bias. Additionally, according to Taiwan National Health Insurance Annual Statistical database, 2010 annual contact rate of outpatient visits (per 100,000 population) under icd-9 code 360-379 based on age distribution were as follows: $32859(0-4$ years), 56,810 (5-9 years), 44,784 (10-14 years), 25,525 (15-19 years), 21,363 (20-24 years), 20,314 (25-29 years), 19,316 (30-34 years), 19,504 (35-39 years),20,770 (40-44 years), 21,695 (45-49 years) and 24,110 (50-54 years) [46]. According to a study, the medical facilities have good accessibility which Taiwanese citizens can see any doctor without a referral [47]. Also, the medical cost is quite low which seeing a doctor is not a huge burden for most citizens. Hence, people in Taiwan tend to seek a professional help when they have any tiny symptoms. Moreover, the data did not include contact rate of inpatient, and icd-9 code 360-379 did not represent all eye diseases. Thus, the true percentage of patients examined by an ophthalmologist is higher than the above data, and the rate of cataracts under-diagnosed is relative low.

\section{Conclusions}

Patients with early-onset cataract have higher risk of developing tinnitus, similar to those of other well-known risk factors in this large population-based study. Of importance, patients should receive further evaluation for early diagnosis and management if any signs of tinnitus occur. Oxidative stress may be one of the main pathogeneses shared by early-onset cataract and tinnitus. Further studies should be conducted to verify whether the relationship is observed only in Asia or worldwide and to confirm the mechanisms involved.

\section{Abbreviations}

HRs: Hazard ratios; aHRs): Adjusted HRs; Cls: Confidence intervals; GSH: Glutathione; UV: Ultraviolet; ROS: Reactive oxygen species;

NHIRD: National Health Insurance Research Database; ICD-9-CM: International Classification of Disease, Ninth Revision, Clinical Modification; DM: Diabetes mellitus

\section{Acknowledgements}

Not applicable.

\section{Authors' contributions}

YYT and CCC designed the work. YCH and CLL were major contributors in writing the initial manuscript. $\mathrm{YCH}$ and $\mathrm{CHC}$ interpreted the patient data. YCH, CLL and CHC contributed to the acquisition and analysis. Critical revision was performed by YCH, TYN, CLL and YYT. CCC and TYN were responsible for supervision. All authors read and approved the final manuscript and agreed to be accountable for all aspects of the work.

\section{Funding}

This work was supported by grants from the Ministry of Health and Welfare, Taiwan (MOHW108-TDU-B-212-133004), China Medical University Hospital, Academia Sinica Stroke Biosignature Project (BM10701010021), MOST Clinical Trial Consortium for Stroke (MOST 107-2321-B-039 -004-), Tseng-Lien Lin Foundation, Taichung, Taiwan, and Katsuzo and Kiyo Aoshima Memorial Funds, Japan. The funding bodies had no role in the design of the study and collection, analysis, and interpretation and data and in the writing of the manuscript.

\section{Availability of data and materials \\ The datasets generated and analysed during the current study are from the National Health Insurance Research Database (NHIRD), which has been transferred to the Health and Welfare Data Science Center (HWDC). Interested researchers can obtain the data through formal application to the HWDC, Department of Statistics, Ministry of Health and Welfare, Taiwan (http://www.nhi.gov.tw/english/index.aspx).}

\section{Ethics approval and consent to participate}

This study was approved by the Institutional Review Board, China Medical University and the Hospital Research Ethics Committee (IRB permit number: CMUH-104-REC2-115). Consent was not obtained from patients, which was approved by the ethical review committee. In addition, no consent was required due to the retrospective nature of this study.

Consent for publication

Consent was not obtained from patients, because the study was a secondary analysis of existing public database. 


\section{Competing interests}

The authors have no conflict of interest.

\section{Author details}

'Department of Ophthalmology, China Medical University Hospital, China Medical University, No. 2, Yude Road, Taichung, Taiwan. ${ }^{2}$ School of Medicine, China Medial University, Taichung, Taiwan. ${ }^{3}$ Management Office for Health Data, China Medical University Hospital, Taichung, Taiwan. ${ }^{4}$ Department of Public Health, China Medical University College of Public Health, Taichung, Taiwan. ${ }^{5}$ Department of Otolarygology, Head and Neck Surgery, China Medical University Hospital, China Medical University, Taichung, Taiwan.

Received: 29 February 2020 Accepted: 1 June 2020

Published online: 16 June 2020

\section{References}

1. World Health Organization. Priority Eye Disease 2. Cataract. https://www. who.int/blindness/causes/priority/en/index1.htm.

2. Joe A. Vinson. Oxidative stress in cataracts. Pathophysiology. 2006;13(3):151-62.

3. Truscott RJ. Age-related nuclear cataract-oxidation is the key. Exp Eye Res. 2005;80:709-25.

4. Tsai SY, Hsu WM, Cheng CY, Liu JH, Chou P. Epidemiologic study of agerelated cataracts among an elderly Chinese population in Shih-Pai Taiwan. Ophthalmology. 2003:110:1089-95.

5. Prchal J, Skalka H, Clements RS, Bradley EL, Conrad ME, Pittman C. Diabetes and risk of cataract development. Metab Pediatr Ophthalmol. 1980;4:185-9.

6. Chen SN, Lin KK, Chao AN, Kuo YH, Ho JD. Nuclear sclerotic cataract in young patients in Taiwan. J Cataract Refract Surg. 2003;29:983-8.

7. Black RL, Oglesby RB, von Sallmann L. Posterior subcapsular cataracts induced by corticosteroids in patients with rheumatoid arthritis. JAMA. 1960;174:166-71

8. Kaluzny JJ. Mean age of patients with senile and presenile cataract in clinical material from 1987-1992. Klin Ocz. 1993:95:277-80.

9. Solberg $Y$, Rosner M, Belkin M. The association between cigarette smoking and ocular diseases. Surv Ophthalmol. 1998;42:535-47.

10. Nemholt SS, Schmidt JH, Wedderkopp N, Baguley DM. Prevalence of tinnitus and/or hyperacusis in children and adolescents: study protocol for a systematic review. BMJ Open. 2015;5:e006649.

11. Langguth B, Kreuzer PM, Kleinjung T, De Ridder D. Tinnitus: causes and clinical management. Lancet Neurol. 2013;12(9):920-30.

12. Han BI, Lee HW, Kim TY, Lim JS, Shin KS. Tinnitus: characteristics, causes, mechanisms, and treatments. J Clin Neurol. 2009;5(1):11-9.

13. Jastreboff PJ, Hazell JW. A neurophysiological approach to tinnitus: clinical implications. Br J Audiol. 1993;27:7-17

14. Haase GM, Prasad KN, Cole WC, Baggett-Strehlau JM, Wyatt SE. Antioxidant micronutrient impact on hearing disorders: concept, rationale, and evidence. Am J Otolaryngol. 2011;32:55-61.

15. Koç S, Akyüz S, Somuk BT, Soyalic H, Yılmaz B, Taskin A, et al. Paraoxonase activity and oxidative status in patients with tinnitus. J Audiol Otol. 2016; 20(1):17-21.

16. Nondahl DM, Cruickshanks KJ, Huang GH, Klein BE, Klein R, et al. Tinnitus and its risk factors in the Beaver dam offspring study. Int J Audiol. 2011;50: 313-20.

17. Congdon N, Vingerling JR, Klein BE, West S, Fried-man DS, Kempen J, et al. Prevalence of cataract and pseudophakia/aphakia among adults in the United States. Arch Ophthalmol. 2004;122:487-94.

18. Kim HJ, Lee HJ, An SY, Sim S, Park B, Kim SW, et al. Analysis of the prevalence and associated risk factors of tinnitus in adults. PLoS One. 2015 10(5):e0127578.

19. Langguth B. A review of tinnitus symptoms beyond 'ringing in the ears': a call to action. Curr Med Res Opin. 2011;27:1635-43.

20. Baguley D, McFerran D, Hall D. Tinnitus. Lancet. 2013;382(9904):1600-7.

21. Dandan Z, Zhigang F, Xinbo G, Wenmin H, Qiongman $Y$, Zhongwen $L$, et al. IIness uncertainty, anxiety and depression in Chineses patients with glaucoma or cataract. Sci Rep. 2018;8:11671.

22. Marilov W, Shorikhina OM. Mental disorders in patients admitted for cataract surgery. Zh Nevrol Psikhiatr Im S S Korsakova. 2009;109:27-30.

23. Klein BE, Cruickshanks KJ, Nondahl DM, Klein R, Dalton DS. Cataract and hearing loss in a population-based study: the Beaver dam studies. Am J Ophthalmol. 2001;132:537-43.
24. Kessel L, Siganos G, Jørgensen T, Larsen M. Sleep disturbances are related to decreased transmission of blue light to the retina caused by lens yellowing. SLEEP. 2011;34(9):1215-9.

25. Alexander I, Cuthbertson FM, Ratnarajan G, Safa R, Mellington FE, Foster RG, et al. Impact of cataract surgery on sleep in patients receiving either ultraviolet blocking or blue-filtering intraocular lens im- plants. Invest Ophthalmol Vis Sci. 2014;55:4999-5004.

26. Giblin FJ. Glutathione: a vital lens antioxidant. J Ocul Pharmacol Ther. 2000; 16:121-35.

27. Boscia F, Grattagliano L, Vandermiale G, Micelli-Ferrari T, Altomare E. The protein oxidation and the lens opacity in humans. Invest Ophthalmol Vis Sci. 2000;41:2461-5.

28. Savastano M, Brescia G, Marioni G. Antioxidant therapy in idiopathic tinnitus: preliminary outcomes. Arch Med Res. 2007;38:456-9.

29. Neri S, Signorelli S, Pulvirenti D, Mauceri B, Cilio D, Bordonaro F, et al. Oxidative stress, nitric oxide, endothelial dysfunction and tin-nitus. Free Radic Res. 2006:40:615-8.

30. Al Samarrai AR, Noor Sunba MS, UI Hassan M. The incidence of unknown diabetes mellitus and impaired glucose tolerance in idiopathic presenile cataract. Dev Ophthalmol. 1989;17:79-81.

31. Fukushima H, Cureoglu S, Schachern PA, Kusunoki T, Oktay MF, Fukushima N. Cochlear changes in patients with type 1 diabetes mellitus. Otolaryngol Head Neck Surg. 2005;133(1):100-6.

32. Kaźmierczak H, Doroszewska G. Metabolic disorders in vertigo, tinnitus, and hearing loss. Int Tinnitus J. 2001;7(1):54-8.

33. Nam SW, Lim DH, Cho KY, Kim HS, Kim K, Chung T-Y. Risk factors of presenile nuclear cataract in health screening study. BMC Ophthalmol. 2018; 18:263.

34. Rahman A, Yahya K, Shaikh A, Fasih U, Zuberi BF. Risk factors associated with pre-senile cataract. Pak J Med Sci. 2011;27(1):145-8.

35. Veile A, Zimmermann $H$, Lorenz $E$, Becher $H$. Is smoking a risk factor for tinnitus? A systematic review, meta-analysis and estimation of the population attributable risk in Germany. BMJ Open. 2018;8(2):e016589 Review.

36. Talhout R, Schulz T, Florek E, van Benthem J, Wester P, Opperhuizen A. Hazardous compounds in tobacco smoke. Int J Environ Res Public Health. 2011;8:613-28

37. Wu R, Wang JJ, Mitchell P, Lamoureux EL, Zheng Y, Rochtchina E, et al. Smoking, socioeconomic factors, and age-related cataract: the Singapore Malay eye study. Arch Ophthalmol. 2010;128:1029-35.

38. Okoliea NP, Audua K. Correlation between cyanide-induced decreases in ocular $\mathrm{Ca}^{2+}$-ATPase and lenticular opacification. JMBR. 2004;3(1):37-41.

39. Little MP, Kitahara CM, Cahoon EK, Bernier MO, Velazquez-Kronen R, Doody $M M$, et al. Occupational radiation exposure and risk of cataract incidence in a cohort of US radiologic technologists. Eur J Epidemiol. 2018;33(12):1179-91.

40. Alberto M, Fabriziomaria G. Cataract frequency and subtypes involved in workers assessed for their solar radiation exposure: a systematic review. Acta Ophthalmol. 2018:96:779-88.

41. Das GK, Boriwal K, Chhabra P, Sahu PK, Kumar S, Kumar N. Presenile cataract and its risk factors: a case control study. J Family Med Prim Care. 2019:8(6): 2120-3.

42. Palmer KT, Griffin MJ, Syddall HE, Davis A, Pannett B, Coggon D. Occupational exposure to noise and the attributable burden of hearing difficulties in Great Britain. Occup Environ Med. 2002;59:634e9.

43. Engdahl B, Krog NH, Kvestad E, Hoffman HJ, Tambs K. Occupation and the risk of bothersome tinnitus: results from a prospective cohort study (HUNT). BMJ Open. 2012:2:e000512.

44. Tadao Y, Masaaki T, Tomoyuki I, Hironao O, Tsutomu. Intratympanic injection of dexamethasone for treatment of tinnitus in patients with sudden sensorineural hearing loss. Audiol Res. 2012;2(1):e2.

45. Shulman A, Goldstein B. Intratympanic drug therapy with steroids for tinnitus control: a preliminary report. Int Tinnitus J. 2000;6(1):10-20.

46. Taiwan Ministry of Health and Welfare. https://dep.mohw.gov.tw/DOS/lp-2 019-113.html.

47. Wu T-Y, Majeed A, Kuo KN. An overview of the healthcare system in Taiwan. London J Prim Care (Abingdon). 2010:3(2):115-9.

\section{Publisher's Note}

Springer Nature remains neutral with regard to jurisdictional claims in published maps and institutional affiliations. 